\title{
RECURRENT HYPONATREMIA AS PRESENTING MANIFESTATION OF PITUITARY MACROADENOMA
}

\author{
R. Rajput, D. Jain and V. Pathak \\ Department of Medicine, Pandit Bhagwat Dayal Sharma University \\ of Health Sciences - India
}

\begin{abstract}
Hyponatremia is commonly seen electrolyte disturbance clinically. It is potentially life-threatening and requires prompt diagnosis and treatment. Non-functioning pituitary macroadenoma generally presents with a headache, visual disturbances, and the diagnosis is often delayed because of nonspecific nature of symptoms. Hyponatremia being initial manifestation before other common symptoms of pituitary macroadenoma is rare. We present a case of 55-year-old male with recurrent episodes of hyponatremia who was found to non-functioning pituitary macroadenoma along with panhypopituitarism. He was managed with hormone replacements. Our case highlights the importance of correct diagnosis of hyponatremia, measurement of the thyroid, adrenal and pituitary function in cases of hyponatremia.
\end{abstract}

Key words: Hyponatremia, pituitary macroadenoma, panhypopituitarism

Corresponding author: Dr. Deepak Jain, Department of Medicine, Pt. B.D. Sharma University of Health Sciences, Rohtak-124001 (Haryana), India, e-mail: jaindeepakdr@gmail.com, Ph: +91-9416147887

\section{INTRODUCTION}

Hyponatremia is one of the most common electrolyte disturbance encountered in hospital admitted patients. It is defined as a serum sodium level less than 135 meq/l. It is basically a disruption of water balance, with a relative excess amount of water in body compared to total sodium and potassium content. It is usually associated with a disturbance in the hormone called antidiuretic hormone (ADH) that governs water balance. Even in disorders related to renal sodium loss, vasopressin activity is generally required for hyponatremia to develop. Recurrent hyponatremia without a definitive diagnosis can be more troublesome to manage because of the need of meticulous use of fluid replacement to avoid neurological complications. It is potentially life threatening if an apt diagnosis is not made and correct treatment is not instituted [1]. Various diseases of the central nervous system can cause hyponatremia, e.g., subarachnoid hemorrhage, severe head injury, pituitary adenoma [2]. Pituitary adenoma to cause hyponatremia before the manifestation of other common symptoms like a headache, decreased vision is unlikely. However, for macroadenoma causing hypopituitarism and hyponatremia directly hormone replacement would suffice. Here we present a case of a male having recurrent episodes of hyponatremia who was eventually diagnosed to have hypopituitarism due to non-functioning macroadenoma.

\section{CASE REPORT}

A 55-year-old male presented to the medical emergency department of PGIMS Rohtak, India with com- 
plaints of fever, vomiting for two days and altered sensorium for one day. The patient was febrile, had pallor with rough, dry and cold skin. Blood pressure was $100 / 70 \mathrm{mmHg}$, pulse rate $66 / \mathrm{min}$ with the respiratory rate of $16 / \mathrm{min}$, JVP was normal, and there was no pedal edema. Cardiovascular, respiratory and abdominal examinations were normal. Central nervous system examination revealed Glasgow Coma Scale score $9 / 15$, the patient had no signs of meningeal irritation, and fundus examination was normal. Investigations showed normal haemogram and urine examination. Serum sodium was $114 \mathrm{mmol} / \mathrm{l}$, potassium $3.8 \mathrm{mmol} / \mathrm{l}$, urea $16 \mathrm{mg} / \mathrm{dl}$, serum creatinine $1.0 \mathrm{mg} / \mathrm{dl}$, and plasma glucose $80 \mathrm{mg} / \mathrm{dl}$. Liver function test and lipid profile were normal. Arterial blood gas analysis revealed $\mathrm{pH}$ of 7.43 , bicarbonate $18 \mathrm{mEq} / \mathrm{l}$, serum anion gap (AG) of $8 \mathrm{mEq} / \mathrm{l}, \mathrm{PaO}_{2} 80$ and $\mathrm{PaCO}_{2} 21 \mathrm{mmHg}$. Serum osmolality was $235 \mathrm{mOsm} / \mathrm{kg}$, urinary osmolality 354 $\mathrm{mOmsm} / \mathrm{kg}$ and urinary sodium was $48 \mathrm{meq} / \mathrm{L}$. CSF analysis, NCCT head, X-ray chest and ECG were normal. The patient was treated with $3 \%$ sodium chloride, and his sensorium improved. On enquiring about previous medical history patient had two similar episodes during last one year for which he was hospitalized and recovered well after treatment. As there were no signs of hypervolemia or hypovolemia a working diagnosis of euvolemic hyponatremia was made, and the patient was further investigated to find out the cause for the recurrent hyponatremia. Thyroid function test and morning serum cortisol were done which showed low T4 with normal TSH and low level of cortisol as shown in table 1. Given low T4 with normal TSH and low cortisol level an MRI pituitary and hypothalamus was done which revealed a well-defined lobulated soft tissue lesion measuring $13.6 \times 16.5 \times 21.5 \mathrm{~mm}$ causing superior displacement of optic chiasma extending into left cavernous sinus with an encirclement of more than $50 \%$ of left internal carotid artery suggestive of the pituitary macroadenoma. Subsequent tests were ordered which showed low levels of ACTH and testosterone. $\mathrm{FSH}, \mathrm{LH}$, and prolactin were in normal range (table 1). Visual field charting was done which showed bitemporal hemianopia. He was diagnosed as a case of panhypopituitarism due non-functioning pituitary macroadenoma with hyponatremia and managed with tablet levothyroxine 75 microgram once daily along with injection hydrocortisone $100 \mathrm{mg}$ eight hourly and dose of testosterone $250 \mathrm{mg}$. During the seven days of stay in hospital patient did not develop hyponatremia and at discharge his sodium level was $137 \mathrm{meq} / \mathrm{l}$. At one month follow-up, the patient is doing well on tablet levothyroxine and tablet hydrocortisone with generalized improvement in wellbeing. The patient was advised to neurosurgery consultation for a decision regarding surgical debulking of the pituitary macroadenoma.

Table 1

\begin{tabular}{|l|c|c|}
\hline Test Name & Patient's Value & Refrence Range \\
\hline T3 & $0.54 \mathrm{ng} / \mathrm{mL}$ & $0.6-1.8 \mathrm{ng} / \mathrm{mL}$ \\
\hline T4 & $4.7 \mathrm{ug} / \mathrm{dL}$ & $5.01-12.45 \mathrm{ug} / \mathrm{dL}$ \\
\hline TSH & $1.51 \mathrm{ulU} / \mathrm{ml}$ & $0.35-5.50 \mathrm{ulU} / \mathrm{ml}$ \\
\hline Cortisol & $3.96 \mu \mathrm{\mu g} / \mathrm{dL}$ & $4.30-22.40 \mathrm{\mu g} / \mathrm{dL}$ \\
\hline ACTH & $27.70 \mathrm{pg} / \mathrm{mL}$ & $<46 \mathrm{pg} / \mathrm{mL}$ \\
\hline Testosterone & $55.65 \mathrm{ng} / \mathrm{dL}$ & $241-827 \mathrm{ng} / \mathrm{dL}$ \\
\hline LH & $1.25 \mathrm{mlU} / \mathrm{mL}$ & $1.50-9.30 \mathrm{mlU} / \mathrm{mL}$ \\
\hline FSH & $19.37 \mathrm{mlU} / \mathrm{mL}$ & $1.40-18.10 \mathrm{mlU} / \mathrm{mL}$ \\
\hline Prolactin & $14 \mathrm{ng} / \mathrm{mL}$ & $2.10-17.70 \mathrm{ng} / \mathrm{mL}$ \\
\hline
\end{tabular}

\section{DISCUSSION}

Hyponatremia is a common electrolyte disturbance and is defined as serum sodium concentration below $135 \mathrm{meq} / \mathrm{L}$. Hyponatremia can result from a wide range of causes. It is of utmost importance to identify the underlying cause of hyponatremia and give a befitting treatment which will prevent its recurrence as severe hyponatremia can lead to altered levels of consciousness, seizures, cerebral edema, coma, and death if not treated correctly. In the majority of subjects who develop hyponatremia renal water ex- cretion is impaired which is usually due to an inability to suppress ADH secretion [3]. Sodium is the principal extracellular fluid cation hence the plasma concentration of sodium determines the serum osmolality. Hypotonic (dilutional) hyponatremia is classified by the extracellular volume status into hypo-, eu- and hypervolemic hyponatremia. Although this classification is useful and fascinating, it should be kept in mind that the clinical assessment of the extracellular fluid volume is difficult in the emergency situation [4]. In our patient being euvolemic with low plasma osmolality and increased urine osmolality a diagnosis 
of SIADH (Syndrome of Inappropriate Anti-diuretic Hormone) seemed most probable. But it is essential to exclude hypothyroidism, hypoadrenalism and diuretic use prior to making a diagnosis of SIADH. Hence, thyroid profile and serum cortisol were done which showed low cortisol level, low T4 level and normal TSH level. In view of above reports the central cause of the hyponatremia was suspected, and MRI pituitary/ hypothalamus was done which showed pituitary macroadenoma. Hyponatremia does occur in patients with hypopituitarism but clinically severe hyponatremia occurring as the presenting manifestation of hypopituitarism due to pituitary macroadenoma is rare. There are only a few cases reported in the literature [5]. Hyponatremia occurring in the setting of a pituitary mass lesion could be due to hypoadrenalism because of ACTH deficiency and hypothyroidism. In secondary adrenal insufficiency due to pituitary adenoma as in our patient, hyponatremia results from impaired excretion of water loads. Cortisol being an inhibitor of ADH secretion, hence low level of cortisol leads to increased ADH secretion and resulting in decreased excretion of water by the kidney and subsequently causing hyponatremia [6]. It has also been shown that loss of suppression of the osmostat for ADH release could be the underlying mechanism for ADH hypersecretion in adrenal insufficiency [7]. Thus, the benefit of corticosteroid replacement is thought to be mainly due to suppression of $A D H$. Hyponatremia is also known to occur with hypothyroidism. The pathophysiological mechanism behind hypothyroidism causing hyponatremia is the inability to excrete free water, and also they do not dilute urine maximally after a water load which could be due to increased ADH levels. Decreased glomerular filtration rate in hypothyroidism which directly diminishes free water excretion by reducing water delivery to the diluting segments can also be responsible for hyponatremia. Regardless of the mechanism, the net effect of the impairment in water excretion is the retention of ingested water and a reduction in the plasma sodium concentration by dilution [8]. Measurement of ADH level in our patient would have substantiated the above hypothesis, but due to resource limitation, it was not done.

Clinically, patients with ACTH deficiency are euvolemic, which can cause clinicians to confuse ACTH deficiency with SIADH. It is essential to rule out hypoadrenalism and hypothyroidism before one makes a diagnosis of SIADH. This may be the case with our patient who was treated earlier for the episode of hyponatremia and correct diagnosis was not made. This highlights the importance of following the specific protocol while making a diagnosis of hyponatremia.
It is often seen that patients with subclinical hypopituitarism develop hyponatremia in the presence of a precipitating factor which could be an infection, surgical procedures, drugs, and fever [9]. Our case apparently had no symptoms related to hypopituitarism like decreased libido, low energy level, and the hypothalamic-pituitary-adrenal axis insufficiency resulted in symptoms only during illness. On questioning, he revealed that during his previous presentations also he had an episode of fever as in this episode.

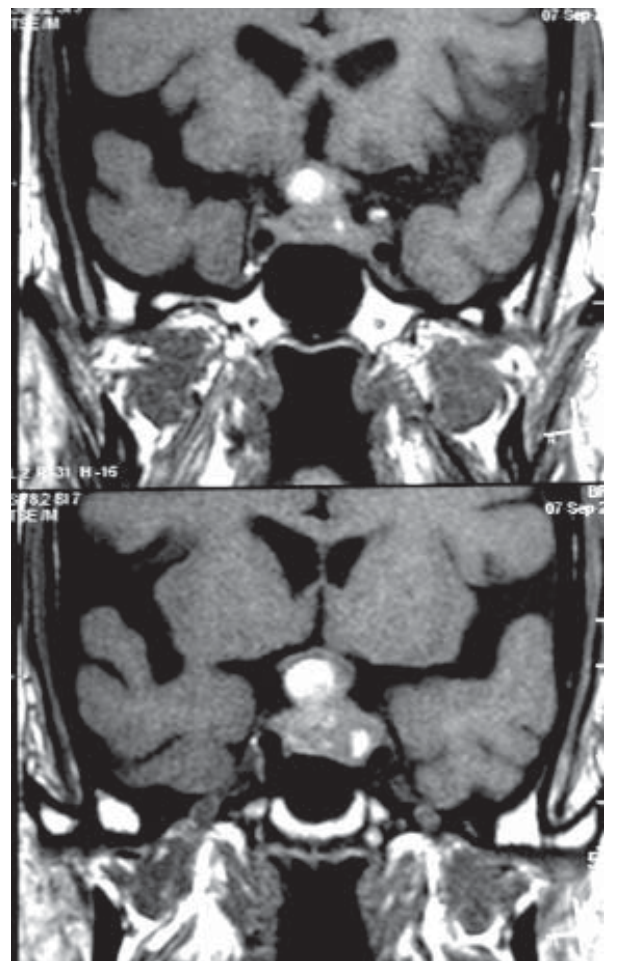

Fig. 1
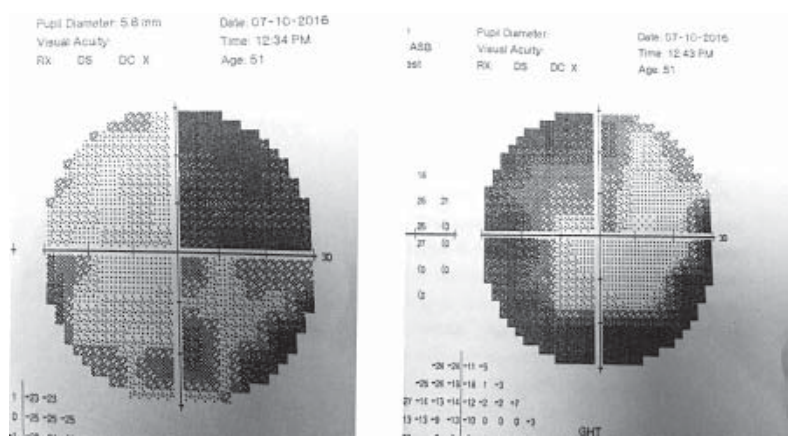

Fig. 2

The intriguing thing about our case is that earlier episodes of hyponatremia were corrected without proper diagnosis and patient recovered well from those episodes with clear fluid and salt replacement. This suggests that apart from dilutional hyponatremia a component of dehydration or sodium deficiency due to salt deficiency may be an 
additional mechanism responsible for the hyponatremia in this case. The suitable treatment of hyponatremia is based on the correct diagnosis of the underlying cause. Approach to hyponatremia could be from a nephrologist's point of view or could be of an endocrinologist. But ultimately both of these approaches emphasize the importance of assessing adequate blood volume status and the measurement of urine sodium concentration. Special consideration should be given to hyponatremia because of CNS disorders which if cured is of prognostic value for the patient. The differentiation between the following conditions: the syndrome of inappropriate antidiuretic hormone secretion (SIADH), acute adrenocorticotropic hormone (ACTH) deficiency, fluid overload and the cerebral salt-wasting syndrome is of utmost importance because management of each differs [10]. Our case shows the various important issues with regard to the workup and management of hyponatremia. The first episode is the time when adequate laboratory studies should be obtained so that a diagnosis can be reached expeditiously. A detailed and conclusive research regarding endocrine control mechanisms causing hyponatremia in the setting of hypopituitarism is lacking and may be a subject of future studies.

\section{CONCLUSION}

Association of hypopituitarism with hyponatremia is clinically relevant as directly hormone replacement will take care of everything and obviates the additional need for painstaking fluid infusion. A minor illness may precipitate severe hyponatremia in hypopituitarism in previously asymptomatic cases, and correct diagnosis can be lifesaving in such situations. Our case highlights the importance of the adrenal, pituitary and thyroid function assessment during evaluation of the recurrent hyponatremia.

\section{REFERENCES}

1. Spasovski G, Vanholder R, Allolio B et al; Hyponatraemia Guideline Development Group. Clinical practice guideline on diagnosis and treatment of hyponatraemia. Eur J Endocrinol. 2014;170(3):G1-47.

2. Yamashiro S, Fuwa I, Seto H, Ushio Y. Initial and postoperative hyponatremia associated with pituitary adenoma:a case report. Acta Neurochir (Wien) 1997;139:987-91.

3. AdroguéHJ, Madias NE Hyponatremia.N Engl $\mathrm{J}$ Med. 2000;342:1581.

4. Thompson C, Hoorn EJ. Hyponatriemia: an overview of frequency, clinical presentation and complications. Clin Endocr Metab 26, 2012, suppl. 1, S1-S6.

5. Lin SH, Hung YH, Lin YF. Severe hyponatremia as the presenting feature of clinically non-functional pituitary adenoma with hypopituitarism. Clin Nephrol2002;57:85-88.

6. Erkut ZA, Pool C, Swaab DF. Glucocorticoids suppress corticotropin-releasing hormone and vasopressin expression in human hypothalamic neurons. J Clin Endocrinol Metab 1998; 83:2066-73.

7. Shibata T, Oeda T, Saito Y. Severe hyponatremia caused by hypothalamic adrenal insufficiency. Intern Med 1999;38:426-32.

8. Hanna FW, Scanlon MF. Hyponatraemia, hypothyroidism, and role of arginine-vasopressin. Lancet 1997; 350:755.

9. Oelkers W. Hyponatremia and inappropriate secretion of vasopressin (antidiuretic hormone) in patients with hypopituitarism. N Engl J Med 1989;321:492.

10. Hanna FW, Scanlon MF. Hyponatraemia, hypothyroidism, and role of arginine-vasopressin. Lancet 1997; 350:755.

11. Thompson C, Berl T, Tejedor A, Johannsson G. Differential diagnosis of hyponatriemia. Clin Endocr Metab 26, 2012, suppl. 1, S7-S15. 